\title{
Data mining, dashboards and statistics: a powerful framework for the chemical design of molecular nanomagnets
}

\section{Yan Duan}

Ben-Gurion University of the Negev https://orcid.org/0000-0002-2849-5602 Joana Coutinho

Centre for Rapid and Sustainable Product Development, Polytechnic of Leiria https://orcid.org/00000002-1571-6904

\section{Lorena Rosaleny}

Instituto de Ciencia Molecular, Universitat de València

\section{Salvador Cardona}

Instituto de Ciencia Molecular-Universitat de València

José J. Baldoví

Instituto de Ciencia Molecular-Universitat de València

\section{Alejandro Gaita-Ariño ( $\nabla$ alejandro.gaita@uv.es )}

Instituto de Ciencia Molecular, Universitat de València https://orcid.org/0000-0002-1600-8627

\section{Article}

Keywords: molecular nanomagnets, data mining

Posted Date: June 1st, 2021

DOl: https://doi.org/10.21203/rs.3.rs-490959/v1

License: (c) (i) This work is licensed under a Creative Commons Attribution 4.0 International License.

Read Full License

Version of Record: A version of this preprint was published at Nature Communications on December 9th, 2022. See the published version at https://doi.org/10.1038/s41467-022-35336-9. 


\section{Abstract}

Three decades of intensive research in molecular nanomagnets have brought the magnetic memory in molecules from liquid helium to liquid nitrogen temperature. The enhancement of this operational temperature relies on a wise choice of the magnetic ion and the coordination environment. However, serendipity, oversimplified theories and chemical intuition have played the main role. In order to establish a powerful framework for statistically driven chemical design, we collected chemical and physical data for lanthanide-based nanomagnets to create a catalogue of over 1400 published experiments, developed an interactive dashboard (SIMDAVIS) to visualise the dataset, and applied inferential statistical analysis to it. We found that the effective energy barrier derived from the Arrhenius equation displays an excellent correlation with the magnetic memory, and that among all chemical families studied, only terbium bisphthalocyaninato sandwiches and dysprosium metallocenes consistently present magnetic memory up to high temperature, but that there are some promising strategies for improvement.

\section{Introduction}

With the goal of facilitating the chemical design of lanthanide single-ion magnets (SIMs), herein we mined experimental data from over 450 articles from the first 17 years of SIM research and applied data visualization tools followed by state-of-the-art statistical analysis. This allowed us to obtain robust statistically-driven insights on the key factors governing the slow relaxation of the magnetisation. Additionally, we created a dataset and a dashboard: a freely accessible online interactive app that allows any user to perform qualitative analyses as well as to browse the dataset. This statistical study represents a quantitative and data-supported verification/refutation of several widely held "chemical intuitions" in the field of molecular magnetism.

\section{A brief history of SIMs}

Molecular nanomagnets were reported for the first time at the beginning of the 1990s, when $\mathrm{Mn}_{12} \mathrm{O}_{12}\left(\mathrm{CH}_{3} \mathrm{COO}\right)_{16}\left(\mathrm{H}_{2} \mathrm{O}\right)_{4}$, a polynuclear magnetic complex with strong magnetic coupling between $d-$ block ions was reported to display magnetic hysteresis, similar to classical magnets, but with a quantum tunnelling mechanism for the relaxation of the magnetisation. ${ }^{1,2}$ This was the first of a plethora of singlemolecule magnets (SMMs), the term coined for systems behaving as hard bulk magnets below a certain temperature, but where the slow relaxation of the magnetisation is of purely unimolecular origin. The magnetic behaviour in SMMs can be approximated to that of an effective anisotropic magnetic moment arising from the exchange interactions between the spins of the metal ions. The reversal of this giant anisotropic spin occurs by populating excited spin states and overcoming an energy barrier. Hence, the thermal dependence of the relaxation rate was described by the Arrhenius equation (see Fig. 1), using this effective energy barrier $\left(U_{\text {eff }}\right)$ and a pre-exponential factor $\left(\tau_{0}\right) .{ }^{3}$ Both parameters were not extracted directly from the hysteresis loop, but rather from the combined frequency- and temperature-dependence of the so-called out-of-phase component of the ac susceptibility ( $\chi^{\prime \prime}$, see Fig. 1). ${ }^{3,4}$ The experimental fact that the magnetic hysteresis was observed only near liquid-helium temperatures was rationalised mainly 
by the typically low values found for the effective energy barrier $U_{\text {eff }} \approx 50 \mathrm{~K} .{ }^{5}$ Initial models based on effective spin Hamiltonians gave rise to the relation $U_{\text {eff }}=D S_{z}{ }^{2}$ and concluded that the best strategy to raise $U_{\text {eff }}$ and, therefore, to improve the maximum hysteresis temperature $\left(T_{\text {hyst }}\right)$ is to maximise the total effective spin $(S)$, rather than the magnetic anisotropy $(D)$. Indeed, the latter is a less straightforward target for the synthetic chemist. ${ }^{6}$ Despite great effort toward the synthesis of such systems and an abundance of molecules with increasing values of $S$, very little progress was made in the first decade in terms of increasing $U_{\text {eff }}$ or $T_{\text {hyst }}{ }^{7}$

In the 2000s, a second generation of molecular nanomagnets emerged, namely bis-phthalocyaninato (Pc) "double deckers". ${ }^{8}$ This novel type of SMMs, commonly known as Single lon Magnets (SIMs), is based on mononuclear complexes containing a single magnetic ion embedded in a coordination environment, thus constituting the smallest molecular nanomagnet. Their properties arise from a strong spin-orbit coupling which, combined with the crystal-field interaction with the surrounding ligands, results in an enhanced magnetic anisotropy when compared to the first generation of SMMs. Identical data treatment using the Arrhenius equation resulted in effective energy barriers $U_{\text {eff }}$ up to an order of magnitude higher for SIMs based on rare-earth ions when compared to those of polynuclear metal complexes of the $d$-block. Similarly, the characteristic maxima in the out-of-phase component of the ac susceptibility $\chi^{\prime \prime}$ moved to higher temperatures, albeit the $T_{\text {hyst }}$ did not increase significantly.

After the germinal $\mathrm{LnPc}_{2}$, different chemical families such as polyoxometalates ${ }^{9}$ and metallocenes ${ }^{10}$ were synthesised and were shown to exhibit slow relaxation of the magnetisation of purely molecular origin (Fig. 2). The realisation that lanthanide SIMs were not restricted to a single chemical strategy inspired a large community of chemists. As a result, in the last 15 years, SIM behaviour was reported in over 600 compounds, whilst above a third of these compounds displayed magnetic hysteresis. Recent efforts have been made to offer some perspective, ${ }^{11-14}$ but so many studies pursuing independent inspirations have been reported that it is hard for any single scientist to distinguish anecdotal claims from proven strategies. Modern techniques of data analysis and visualization can contribute to remedy this knowledge gap. In particular, dashboards are intuitive graphical software applications for dynamic data visualization and information management, of growing popularity in different fields. ${ }^{15-17}$

The present work aims to rationalize the correlations among the different physical variables involved in SIMs. A common working hypothesis is that the parameters arising from the ac magnetometry (e.g. $\left.U_{\text {eff }}\right)$ are well correlated with the experimental values (e.g. $\left.T_{\text {hyst }}\right)$. This, however, has not been proven and has actually been challenged in various ways. ${ }^{14,28,29}$ Over the years, various theoretical approaches have put the focus on the role of different physical processes and different parameters. ${ }^{28-31}$ In order to provide the synthetic chemists with a statistically-driven chemical design guide, we applied the techniques of third generation computational chemistry, ${ }^{32}$ starting by collecting a high-quality dataset and representing the data in an interactive dashboard. 


\section{Results}

\section{An interactive dashboard for lanthanide SIMs}

In this work, we built a dataset of the most relevant chemical and physical properties of 1405 lanthanide SIM samples collected from 451 scientific articles (Supplementary Section 7) published between 2003 and 2019 and developed a user-friendly dashboard-style web application named SIMDAVIS (Single lon Magnet DAta VISualization) to host it. The dataset contains over 10000 independent pieces of chemical information, as well as over 5000 independent pieces of physical (magnetic) information. Furthermore, the dataset is hierarchically clustered into magnetostructural "taxonomies" (see Supplementary Sections 4 and 6) in order to pave the way for further analysis, including Machine Learning studies.

SIMDAVIS allows the chemical community to visualize the key relationships between chemical structures and physical properties in our catalogue of SIMs. Our interactive dashboard can be directly invoked by accessing the internet site where it is located. ${ }^{33}$ It is organized in 6 main tabs: Home, ScatterPlots, BoxPlots, BarCharts, Data (View Data and Download Data) and About SIMDAVIS (Variables, Authors, Feedback\&Bugs, Changelog and License) as we can observe in Fig. 3.

In the SIMDAVIS dashboard, the most versatile source of graphical information is the "ScatterPlots" tab, where an example plot is explained in Fig. 3. The next two tabs display the data in complementary ways. The "BoxPlots" tab allows to examine the distribution of each SIMs quantitative property vs a categorization criterion, e.g. we can see the distribution of $U_{\text {eff }}$ values as a function of the coordination elements. The boxplot for each category is shown, including the median and the interquartile range. The "BarCharts" tab allows the exploration of the frequency of different qualitative variables in our dataset. Stacked bar graphs allow the simultaneous analysis of two qualitative variables, e.g. we can display, for each chemical family, the number of samples which present magnetic hysteresis. The "Data" tab is a powerful interface to browse the dataset, featuring the possibility to choose the data columns to show, ordering in ascending or descending order, and filtering by arbitrary keywords; it also permits downloading all data, including links to the CIF files, when available. Finally, the "About SIMDAVIS" tab contains information about the variables contained in the dataset.

\section{Statistically-driven chemical design of SIMs}

SIMDAVIS allows the visualization of the relationships between chemical and physical variables in SIMs, and thereby enables determining the main variables that the synthetic chemist needs to consider to obtain the desired physical properties. We will first analyze this qualitatively employing a series of boxplots, violin plots and bar charts (see Fig. 4 and Supplementary Figs. 11.1-11.6, 12). The full statistical analysis is presented in Supplementary Sections 4, 5 and 6.

First, let us focus on the effective energy barrier $U_{\text {eff }}$ and the blocking temperature $T_{\mathrm{B} 3}$ (the temperature for maximum out-of-phase ac susceptibility $\chi^{\prime \prime}$ at $10^{3} \mathrm{~Hz}$, see Fig.1). From Fig. 4 and Supplementary Figs. 11.1-11.4, we can see that the only chemical family with a clearly distinct behaviour is the $\mathrm{LnPc}_{2}$ family, 
with median values of $U_{\text {eff }}>300 \mathrm{~K}$ and $T_{\mathrm{B} 3}>30 \mathrm{~K}$. Equivalently, one can see that $\mathrm{Dy}{ }^{3+}$ and $\mathrm{Tb}^{3+}$ are somewhat better than the rest, and that in general oblate ions perform better than prolate ions, for both properties. In addition, non-Kramers ions present higher median $T_{\mathrm{B} 3}$ but similar $U_{\text {eff }}$ values compared with Kramers ions.

Now, let us analyse the maximum hysteresis temperature $T_{\text {hyst }}$ which has been much less studied despite being the main justification for this whole field. The only chemical family with a distinct positive behaviour is the metallocene family. More surprisingly, $\mathrm{Er}^{3+}$ complexes have distinctly high hysteresis temperatures, markedly with a higher median than $\mathrm{Dy}^{3+}$ or $\mathrm{Tb}^{3+}$ complexes. This is in sharp contrast with their relative $T_{\mathrm{B} 3}$ values which are consistently much lower in the case of $\mathrm{Er}^{3+}$ complexes. This not only indicates that searching for equatorial environments, precisely the ones that favour good magnetic properties in $\mathrm{Er}^{3+}$ complexes, ${ }^{30}$ often results in more rigid ligands, but also indicates an underexplored territory. It is certainly possible that chemical modifications of $\left[\mathrm{Er}(\mathrm{COT})_{2}\right]^{-}$(or other $\mathrm{Er}^{3+}$ record-bearing complexes) designed to optimise the detrimental effect of molecular vibrations may achieve records that are competitive with $\mathrm{DyCp}_{2}$. Prolate ions are consistently -and surprisingly-better than the oblate ones, having a higher median value for $T_{\text {hyst }}$. This is again in contrast with the opposite behaviour which is observed for $T_{\mathrm{B} 3}$ and $U_{\text {eff, }}$ and possibly again due to the influence of $\mathrm{Er}^{3+}$ complexes with their more rigid equatorial environments. Finally, the coordination number and the number of ligands do have an influence on the statistically expected hysteresis temperature, with the best ones being 2 and 7 in the case of the coordination number and just 7 for the number of ligands. As we will discuss below, there are chemical insights to be gained from this.

To put all these trends into perspective, it is important to numerically analyse the connexion between the different variables and the clustering of our data. A lognormal analysis (see Supplementary Section 4.3) shows that the three main chemical variables, namely the chemical family, the lanthanide ion and the coordination elements, are sufficient to reasonably explain the variation of values of the others, meaning there is a limit on the information one can independently extract from the rest of the chemical variables. Multiple correspondence analysis (see Supplementary Sections 4.1,4.2) suggests a chemical clustering that consists in three small groups, namely $\mathrm{Gd}^{3+}$ complexes, metallocenes and $\mathrm{LnPc}_{2}$ double deckers, and two much larger groups with a large overlap with oblate and prolate ions respectively. A factorial analysis of mixed data considering also all magnetic information available (see Supplementary Section 6) simplifies the clustering to three groups. Again, the two distinct families present a large overlap with metallocenes and $\mathrm{LnPc}_{2}$ double decker chemical families, both of them presenting significantly better properties than the other kinds of samples.

Further insight is provided by bar charts representing the reported presence of magnetic hysteresis, whether full or pinched, as a function of different chemical variables (Fig. 4). Note that we are limited by the minority of the samples where hysteresis or its absence is reported; in the vast majority of the cases 
this information is lacking. Nevertheless, here it is apparent that certain families such as $\operatorname{LnPc}_{2}(\mathrm{and}$ metallocenes) tend to display (pinched) hysteresis.

\section{The effective energy barrier: oversimplified yet meaningful}

A key question is how much the analyses in this field have been affected by the simplified assumption that SIMs relax via an Orbach mechanism, which is characterised by $\tau_{0}$ and $U_{\text {eff. It has been pointed out }}$ that frequently, as $U_{\text {eff }}$ increases, $\tau_{0}$ decreases, leaving relaxation times essentially constant. ${ }^{5}$ The classical text of Abragam and Bleaney offered the following relation between the two parameters for the two-phonon Orbach process: ${ }^{34}$

$$
\frac{1}{\tau_{0}}=R_{\mathrm{Or}} \cdot\left(U_{\mathrm{eff}}\right)^{n}
$$

where $n=3$, reasonable parameters for rare earth elements resulted in an Orbach rate $R_{\mathrm{Or}} \approx 10^{4} \mathrm{~K}^{-3} \cdot \mathrm{s}^{-1}$, and early experimental results were in the range $10^{3} \mathrm{~K}^{-3} \cdot \mathrm{s}^{-1}<R_{\mathrm{Or}}<10^{5} \mathrm{~K}^{-3} \cdot \mathrm{s}^{-1}$. Fitting $\tau_{0} v s U_{\text {eff }}$ in our dataset to equation ( 1 ) results in $n \approx 2.4, R_{\mathrm{Or}} \approx 10^{3} \mathrm{~K}^{-3} \mathrm{~s}^{-1}$ (see Supplementary Table 5). This minor discrepancy with the expected exponent serves as an independent evaluation of the limitations of a simple Orbach model. We also find $R_{\text {or }}$ (prolate) $\approx 5 \cdot R_{\text {or }}$ (oblate), meaning that, for comparable $U_{\text {eff, }}$ relaxation for oblate ions is on average substantially slower than that for prolate ions. This is consistent with the observation that complexes of oblate ions present values of $T_{\mathrm{B} 3}$ higher than expected considering their $U_{\text {eff }}$ (see Supplementary Fig. 11.3). The limited (<100) data points of $U_{\text {eff,ff, }} \tau_{0, f f}$ pairs, where all relaxation processes were considered, present a better agreement on the exponent, with $n \approx 3$ and lower Orbach rates $R_{\mathrm{Or}} \approx 150 \mathrm{~K}^{-3} \mathrm{~s}^{-1}$.

The remaining crucial issue is to quantify up to what level the value of $U_{\text {eff }}$ and $\tau_{0}$ are well correlated with the slow relaxation of the magnetisation, or to determine whether one would need to employ $U_{\text {eff,ff }}$ instead. Let us proceed with increasing the order of complexity. A visual inspection in SIMDAVIS shows that, in a few cases where there is simultaneous information on $U_{\text {eff }}$ and $U_{\text {eff,ff, }}$, their values are very similar (Fig. 5a). Furthermore, this partial information is corroborated by the very similar dependencies of $T_{\mathrm{B} 3}$ or

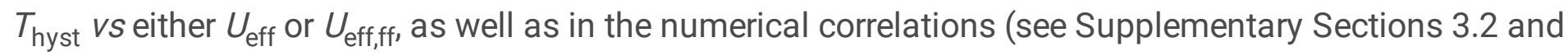
5.3). A categorical analysis (Figs. 5b, c) shows that the data dispersion is large, meaning that it is impossible to predict the experimental behaviour for an individual sample merely from its $U_{\text {eff }}$ value. However, it demonstrates that, statistically, samples which present a maximum in the out-of-phase susceptibility $\chi^{\prime \prime}$, or hysteresis, also present higher $U_{\text {eff }}$ values. A more thorough numerical analysis (see Supplementary Section 6) confirms these trends.

An in-depth statistical analysis of all physical parameters based on the Akaike Information Criterion (see Supplementary Section 5.3) concludes that $U_{\text {eff }}$ derived from a simple Arrhenius plot is the best single 
predictor for the magnetic behaviour in our dataset. This means that, whether we are discussing in terms of the out-of-phase component of the ac susceptibility or magnetic hysteresis, $U_{\text {eff }}$ is a better predictor than $\tau_{0}, \tau_{0, f f}, U_{\text {eff,2 }}$ and, in practice, than $U_{\text {eff,ff. }}$. Factorial analysis of mixed data (see Supplementary Section 6) also reveals the predictive power of $U_{\text {eff }}$ compared with $\tau_{0}$. Note that this does not contradict previous studies which demonstrated that a variation in the Orbach barrier does not fully explain the differences in retention of magnetisation, ${ }^{14}$ since we have not explicitly considered other relaxation mechanisms in the present work.

\section{Conclusions}

We have systematically analysed over 450 articles to collect information from over 1400 samples reported over the first 17 years of the field of lanthanide-based SIMs and built a user-friendly dashboard for the visualization of all the collected data. Moreover, we carried out an in-depth statistical analysis that allowed extracting trends, distinguishing the most relevant variables and grouping the data in clusters based on their chemical and physical properties. From this study, we can highlight two main pieces of information.

In the first place, from the point of view of the parametric characterisation, the simple Arrhenius fit assuming an Orbach process has been proven to be surprisingly meaningful, with the expected relation between $\tau_{0}$ and $U_{\text {eff }}$. One can therefore perform this oversimplified theoretical fit knowing that the effective energy barrier $U_{\text {eff }}$ has been proven to present a consistently good correlation with SMM behaviour. Crucially, we have also shown the very different nature of short-term magnetic memory in form of the blocking temperature $T_{\mathrm{B} 3}$ at $10^{3} \mathrm{~Hz}$ and its long-term counterpart in the form of maximum hysteresis temperature $T_{\text {hyst }}$. The best strategies that optimize the former are not necessarily the best for the latter.

In the second place, the chemical roadmap for the preparation of lanthanide complexes with higher $T_{\text {hyst }}$ becomes now a little clearer. There is a single chemical strategy that consistently produces good magnetic memories, namely sandwiching an oblate ion between two rigid, planar, aromatic ligands; furthermore, the ion should be chosen to result in the most favourable $M_{\mathrm{J}}$ structure, given the electron distribution offered by the ligand. Up to now, only two chemical families are well adapted to this strategy, namely $\mathrm{TbPc}_{2}$ complexes and dysprosium metallocenes. Optimization is ongoing within these two families, for example $\mathrm{TbPc}_{2}$ complexes featuring a radical Pc display enhanced properties, ${ }^{35}$ and the reduced (divalent) analogues of $\mathrm{DyCp}_{2} .{ }^{36}$ We find comparatively little value in further pursuing chemical strategies that have been amply explored and never yielded hysteresis above $10 \mathrm{~K}$. On the other hand, we also evidence that there is, of course, value in chemical ingenuity and exploration, in the quest for another successful strategy, which according to our results might well be based on equatorial erbium complexes, since these display consistently high $T_{\text {hyst }}$ values. Note that a few complexes included in our data fall into ample families such as "mixed ligands" or "other families", and yet present excellent hysteresis 
temperatures. It is possible that the next family of record-setters is related to one of the promising candidates in Fig. 6. Two axial phosphine oxide ligands with bulky substituents seem to function in a similar way as metallocenes, despite the five equatorial $\mathrm{H}_{2} \mathrm{O}$ molecules. ${ }^{24,37}$ This strategy is not restricted to phosphine oxides and deserves to be explored further: as can be seen in Supplementary Fig. 11.4, complexes with 7 ligands have median values of $T_{\text {hyst }}$ close to $10 \mathrm{~K}$, as high as those with 2 ligands.

At the same time, here we provide a catalogue of lanthanide SIMs, together with SIMDAVIS, a dashboard that allows its interactive navigation; this is a type of tool utterly missing in the field of molecular nanomagnets. Perhaps more importantly in the wider perspective of design of new materials ${ }^{38-40}$ and new molecules, ${ }^{32,41}$ the dataset curated in this work will serve for Machine Learning studies and can also be employed as an annotated training data set for the development of new web scraping systems to retrieve chemical data, ${ }^{42,43}$ or even word embeddings, ${ }^{44}$ from the scientific literature. Finally, this work constitutes a step towards the availability of findable, accessible, interoperable, and reusable (FAIR) data in Chemistry. ${ }^{45}$

\section{Methods}

Data gathering. This process started with the collection and organisation of literature data. The following search criterion was applied for the manuscript: articles are searched via Web of Science, employing this code:

TOPIC: TS=((lanthan* OR 4\$f OR "rare\$earth") AND ((single NEAR/1 magnet*) OR "slow relaxation")) Timespan: 2003-2019

For an article to be included in the study, it needs to contain data on at least one compound with certain requirements as follows: (a) contain one trivalent lanthanide ion from the set $\mathrm{Ln}=\{\mathrm{Pr}, \mathrm{Nd}, \mathrm{Sm}, \mathrm{Gd}, \mathrm{Tb}, \mathrm{Dy}$, $\mathrm{Ho}, \mathrm{Er}, \mathrm{Tm}, \mathrm{Yb}$ \} and (b) contain no other paramagnetic entity with the only accepted exception being the presence of a single radical in the coordination sphere and (c) present no strong Ln-Ln interaction, in particular meaning the Ln-Ln distance needs to be larger than $5 \AA$ and more than 3 bridging atoms between neighbouring $L n$ centres, and there cannot be a radical in the bridge. Additionally, the data needs to include at least one of the following information: (a) whether $\chi^{\prime \prime}$ presents a maximum as a function of $T$, or a mere frequency-dependence, or neither; (b) $\chi^{\prime \prime} v s T$ with at least one frequency $(f)$ in the window 0.9 $\mathrm{kHz} \leq f \leq 1.1 \mathrm{kHz}$ and at a field $(B)$ in the window $0 \leq B \leq 2 \mathrm{~T}$; (c) $U_{\text {eff; }}$ (d) the presence or absence of hysteresis; (e) $T_{\text {hyst }}$ at sweep speeds ( $v$ ) in the window $0.05 \mathrm{~T} / \mathrm{s} \leq v \leq 0.3 \mathrm{~T} / \mathrm{s}$. The compounds were classified in chemical families: $\mathrm{LnPc}_{2}$, polyoxometalates, Schiff base, metallocenes, diketonates, radicals, TM near Ln, mixed ligands, and other families. Furthermore, we registered for each sample (when available), the lanthanide ion, its concentration, the coordination number and number of ligands coordinated to the lanthanide ions, the coordination elements, the presence of a field-dependent $\chi^{\prime \prime}$ or a maximum, the temperature of said maximum in presence or absence of an external magnetic field, the external magnetic field, the extracted effective energy barrier and relaxation time, either from a simplified 
Arrhenius fit or from a model considering all relaxation processes, whether these were extracted from the maxima of $\chi^{\prime \prime} v s \mathrm{~T}$ at different frequencies or from an Argand fit, the presence of hysteresis in the magnetisation, and the maximum temperature at which it was recorded. Additionally the DOI, the full reference to the original article, and a link to a CIF file were recorded for each sample. Further details including the classification in chemical families and the criteria for data extraction are provided in Supplementary Sections 1 and 2.

SIMDAVIS dashboard. We programmed the dashboard using R language ${ }^{51,52}$ and shiny, ${ }^{53}$ an open source $\mathrm{R}$ package to create the interactive web app. The design aimed to obtain a clean and simple user interface that adapts automatically to any screen size. The R packages readr, ${ }^{54} \mathrm{dplyr},{ }^{55} \mathrm{DT},{ }^{56}$ ggplot2 ${ }^{57}$ and rcrossref ${ }^{58}$ were also employed in the development of the dataset or the app. The dashboard-style web application is available at https://go.uv.es/rosaleny/SIMDAVIS. This interface allows for variables in the analysis, and subsets of the data, to be adjusted and chosen in real time.

Statistical analysis. The statistical analysis was also based on $\mathrm{R}$, a widely used software environment for statistical computing and graphics, and included the Gifi system for Multiple Correspondence Analysis ${ }^{59}$ ( $\mathrm{R}$ homals package, ${ }^{60}$ ade4 package, ${ }^{61}$ see details in Supplementary Section 4.1), hierarchical clustering studies (FactoMineR, ${ }^{62}$ see details in Supplementary Section 4.2), lognormal modelling (Poisson's distribution, see Supplementary Section 4.3), factorial analysis of mixed data (FactoMineR and factoextra, ${ }^{63}$ see details in Supplementary Section 6) as well as Pearson's product-moment correlation and the Akaike information criterion (AIC) ${ }^{64}$ (see details in Supplementary Section 5.3). The analysis was repeated and verified an overall excellent qualitative and quantitative consistency in all results between the period 2003-2017 (1044 samples) and 2003-2019 (1405 samples).

\section{Declarations}

\section{Data and code availability}

The dataset collected and analysed during this study is freely available for download at https://go.uv.es/rosaleny/SIMDAVIS.

All custom code generated and employed for this study, namely the SIMDAVIS app version 1.1.6, is freely available for download at https://bitbucket.org/rosaleny/simdavis/src/master/.

\section{Acknowledgements}

This work has been supported by the COST Action MolSpin on Molecular Spintronics (Project 15128), H2020 (FATMOLS project) and QUANTERA (SUMO project), the European Research Council (ERC) under the European Union's Horizon 2020 research and innovation programme (grant agreement No 647301 for CoG "DECRESIM"), the Spanish MINECO (grants MAT2017-89993-R and CTQ2017-89528-P cofinanced by FEDER and Excellence Unit María de Maeztu CEX2019-000919-M), the Fundação para a Ciência e a Tecnologia, projects UIDB/04044/2020 and UIDP/04044/2020, and the Generalitat Valenciana (Prometeo 
Program of Excellence and CDEIGENT/2019/022). The statistical analysis was performed by Raquel Gavidia Josa with the Statistical Section of the S.C.S.I.E. (Universitat de València). TOC figure created with BioRender.com.

\section{Author contributions}

All authors contributed to the different stages of the work plan as detailed below.

A.G.A. suggested the starting point of the analysis, with contributions from J.J.B. and S.C.S.

J.C. and Y.D. designed the whole procedure for raw data extraction and classification.

Y.D., J.C., A.G.A., J.J.B. and S.C.S. did the manual data-mining. Y.D., J.C., A.G.A, L.E.R. and S.C.S. doublechecked the raw data.

L.E.R. and A.G.A. cleaned and organized the raw data into a tidy dataset.

L.E.R. and A.G.A. conceived and A.G.A. supervised the statistical data analysis.

L.E.R. conceived and programmed the dashboard-style interactive web application for data visualization and analysis.

All authors contributed to the preparation of the manuscript.

\section{Competing interests}

The authors declare no competing interests.

\section{Additional information}

Supplementary information is available for this paper.

Correspondence should be addressed to J.T.C, L.E.R., A.G.A.

Y.D., J.T.C., L.E.R. contributed equally.

\section{References}

1. Christou, G., Gatteschi, D., Hendrickson, D. N. \& Sessoli, R. Single-molecule magnets. MRS Bull. 25, 66-71 (2000).

2. Sessoli, R., Gatteschi, D., Caneschi, A. \& Novak, M. A. Magnetic bistability in a metal-ion cluster. Nature 365, 141-143 (1993).

3. Villain, J., Hartman-Boutron, F., Sessoli, R. \& Rettori, A. Magnetic relaxation in big magnetic molecules. Europhys. Lett. 27, 159-164 (1994). 
4. Novak, M. A., Sessoli, R., Caneschi, A. \& Gatteschi, D. Magnetic properties of a Mn cluster organic compound. J. Magn. Magn. Mater. 146, 211-213 (1995).

5. Benelli, C. \& Gatteschi, D. Introduction to Molecular Magnetism. From Transition Metals to Lanthanides. (Wiley-VCH, Weinheim, 2015).

6. Gatteschi, D. \& Sessoli, R. Quantum tunneling of magnetization and related phenomena in molecular materials. Angew. Chem. Int. Ed. 42, 268-297 (2003).

7. Milios, C. J. et al. A record anisotropy barrier for a single-molecule magnet. J. Am. Chem. Soc. 129, 2754-2755 (2007).

8. Ishikawa, N., Sugita, M., Ishikawa, T., Koshihara, S. \& Kaizu, Y. Lanthanide double-decker complexes functioning as magnets at the single-molecular level. J. Am. Chem. Soc. 125, 8694-8695 (2003).

9. AlDamen, M. A., Clemente-Juan, J. M., Coronado, E., Martí-Gastaldo, C. \& Gaita-Ariño, A. Mononuclear lanthanide single-molecule magnets based on polyoxometalates. J. Am. Chem. Soc. 130, 88748875 (2008).

10. Jiang, S.-D., Wang, B.-W., Sun, H.-L., Wang, Z.-M. \& Gao, S. An organometallic single-ion magnet. J. Am. Chem. Soc. 133, 4730-4733 (2011).

11. McAdams, S. G., Ariciu, A.-M., Kostopoulos, A. K., Walsh, J. P. S. \& Tuna, F. Molecular single-ion magnets based on lanthanides and actinides: Design considerations and new advances in the context of quantum technologies. Coord. Chem. Rev. 346, 216-239 (2017).

12. Dey, A., Kalita, P. \& Chandrasekhar, V. Lanthanide(III)-based single-ion magnets. ACS Omega 3, 94629475 (2018).

13. Feng, M. \& Tong, M.-L. Single ion magnets from $3 d$ to $5 f$. developments and strategies. Chem. - Eur. J. 24, 7574-7594 (2018).

14. Giansiracusa, M. J., Kostopoulos, A. K., Collison, D., Winpenny, R. E. P. \& Chilton, N. F. Correlating blocking temperatures with relaxation mechanisms in monometallic single-molecule magnets with high energy barriers $\left(U_{\text {eff }}>600 \mathrm{~K}\right)$. Chem. Commun. 55, 7025-7028 (2019).

15. Mills, M. C. \& Rahal, C. The GWAS Diversity Monitor tracks diversity by disease in real time. Nat. Genet. 52, 242-243 (2020).

16. Fanzo, J. et al. The Food Systems Dashboard is a new tool to inform better food policy. Nat. Food 1, 243-246 (2020).

17. Dong, E., Du, H. \& Gardner, L. An interactive web-based dashboard to track COVID-19 in real time. Lancet Infect. Dis. 20, 533-534 (2020).

18. Horii, Y., Katoh, K., Breedlove, B. K. \& Yamashita, M. Elongation of magnetic relaxation times in a single-molecule magnet through intermetallic interactions: a clamshell-type dinuclear terbium(III)phthalocyaninato quadruple-decker complex. Chem. Commun. 53, 8561-8564 (2017).

19. Ishikawa, N. et al. Upward temperature shift of the intrinsic phase lag of the magnetization of bis(phthalocyaninato)terbium by ligand oxidation creating an $S=1 / 2$ spin. Inorg. Chem. 43, 54985500 (2004). 
20. Goodwin, C. A. P., Ortu, F., Reta, D., Chilton, N. F. \& Mills, D. P. Molecular magnetic hysteresis at 60 kelvin in dysprosocenium. Nature 548, 439-442 (2017).

21. Guo, F.-S. et al. Magnetic hysteresis up to 80 kelvin in a dysprosium metallocene single-molecule magnet. Science 362, 1400-1403 (2018).

22. McClain, K. R. et al. High-temperature magnetic blocking and magneto-structural correlations in a series of dysprosium(III) metallocenium single-molecule magnets. Chem. Sci. 9, 8492-8503 (2018).

23. Liu, J.-L. et al. Switching the anisotropy barrier of a single-ion magnet by symmetry change from quasi- $D_{5 \mathrm{~h}}$ to quasi- $O_{\mathrm{h}}$. Chem. Sci. 4, 3310-3316 (2013).

24. Gupta, S. K., Rajeshkumar, T., Rajaraman, G. \& Murugavel, R. An air-stable Dy(III) single-ion magnet with high anisotropy barrier and blocking temperature. Chem. Sci. 7, 5181-5191 (2016).

25. Poneti, G. et al. A rational approach to the modulation of the dynamics of the magnetisation in a dysprosium-nitronyl-nitroxide radical complex. Chem. Commun. 1807-1809 (2007).

26. Demir, S., Jeon, I.-R., Long, J. R. \& Harris, T. D. Radical ligand-containing single-molecule magnets. Coord. Chem. Rev. 289-290, 149-176 (2015).

27. Sun, W.-B. et al. The slow magnetic relaxation regulated by ligand conformation of a lanthanide single-ion magnet $\left[\mathrm{Hex}_{4} \mathrm{~N}\right]\left[\mathrm{Dy}(\mathrm{DBM})_{4}\right]$. Inorg. Chem. Front. 1, 503-509 (2014).

28. Escalera-Moreno, L., Baldoví, J. J., Gaita-Ariño, A. \& Coronado, E. Spin states, vibrations and spin relaxation in molecular nanomagnets and spin qubits: a critical perspective. Chem. Sci. 9, 32653275 (2018).

29. Lunghi, A., Totti, F., Sessoli, R. \& Sanvito, S. The role of anharmonic phonons in under-barrier spin relaxation of single molecule magnets. Nat. Commun. 8, 14620 (2017).

30. Rinehart, J. D. \& Long, J. R. Exploiting single-ion anisotropy in the design of $f$-element singlemolecule magnets. Chem. Sci. 2, 2078-2085 (2011).

31. Baldoví, J. J. et al. Rational design of single-ion magnets and spin qubits based on mononuclear lanthanoid complexes. Inorg. Chem. 51, 12565-12574 (2012).

32. Butler, K. T., Davies, D. W., Cartwright, H., Isayev, O. \& Walsh, A. Machine learning for molecular and materials science. Nature 559, 547-555 (2018).

33. SIM DAtaset VISualisation. https://go.uv.es/rosaleny/SIMDAVIS.

34. Abragam, A. \& Bleaney, B. Electron Paramagnetic Resonance of Transition Ions. (Oxford University Press, Oxford, New York, 2012).

35. Komijani, D. et al. Radical-lanthanide ferromagnetic interaction in a $\mathrm{Tb}(\mathrm{III})$ bis-phthalocyaninato complex. Phys. Rev. Mater. 2, 024405 (2018).

36. Gould, C. A. et al. Synthesis and magnetism of neutral, linear metallocene complexes of terbium(II) and dysprosium(II). J. Am. Chem. Soc. 141, 12967-12973 (2019).

37. Chen, Y.-C. et al. Symmetry-supported magnetic blocking at $20 \mathrm{~K}$ in pentagonal bipyramidal Dy(III) single-ion magnets. J. Am. Chem. Soc. 138, 2829-2837 (2016). 
38. Draxl, C. \& Scheffler, M. NOMAD: The FAIR concept for big data-driven materials science. MRS Bull. 43, 676-682 (2018).

39. de Pablo, J. J. et al. New frontiers for the materials genome initiative. Npj Comput. Mater. 5, 1-23 (2019).

40. Zhang, T. et al. Catalogue of topological electronic materials. Nature 566, 475-479 (2019).

41. Duros, V. et al. Intuition-enabled machine learning beats the competition when joint human-robot teams perform inorganic chemical experiments. J. Chem. Inf. Model. 59, 2664-2671 (2019).

42. Swain, M. C. \& Cole, J. M. ChemDataExtractor: A toolkit for automated extraction of chemical information from the scientific literature. J. Chem. Inf. Model. 56, 1894-1904 (2016).

43. Krallinger, M., Rabal, O., Lourenço, A., Oyarzabal, J. \& Valencia, A. Information retrieval and text mining technologies for chemistry. Chem. Rev. 117, 7673-7761 (2017).

44. Tshitoyan, V. et al. Unsupervised word embeddings capture latent knowledge from materials science literature. Nature 571, 95-98 (2019).

45. Wilkinson, M. D. et al. The FAIR guiding principles for scientific data management and stewardship. Sci. Data 3, 160018 (2016).

46. Huang, W. et al. Metallogrid single-molecule magnet: solvent-induced nuclearity transformation and magnetic hysteresis at $16 \mathrm{~K}$. Inorg. Chem. 55, 5476-5484 (2016).

47. Gregson, M. et al. A monometallic lanthanide bis(methanediide) single molecule magnet with a large energy barrier and complex spin relaxation behaviour. Chem. Sci. 7, 155-165 (2015).

48. Liu, J. et al. A stable pentagonal bipyramidal Dy(III) single-ion magnet with a record magnetization reversal barrier over 1000 K. J. Am. Chem. Soc. 138, 5441-5450 (2016).

49. Harriman, K. L. M., Brosmer, J. L., Ungur, L., Diaconescu, P. L. \& Murugesu, M. Pursuit of record breaking energy barriers: a study of magnetic axiality in diamide ligated Dy ${ }^{\text {III }}$ single-molecule magnets. J. Am. Chem. Soc. 139, 1420-1423 (2017).

50. Wu, J. et al. Cis-trans isomerism modulates the magnetic relaxation of dysprosium single-molecule magnets. Chem. Sci. 7, 3632-3639 (2016).

51. R Core Team. R: A language and environment for statistical computing. R version 3.4.4. https://www.R-project.org/ (R Foundation for Statistical Computing, Vienna, Austria, 2018).

52. RStudio Team. RStudio: Integrated Development Environment for R. Version 1.1.456 http://www.rstudio.com (RStudio Inc., 2018).

53. Chang, W., Cheng, J., Allaire, J. J., Xie, Y. \& McPherson, J. shiny: Web application framework for R. R package version 1.4.0. https://CRAN.R-project.org/package=shiny (2019).

54. Wickham, H. et al. readr: Read rectangular text data. R package version 1.3.1. https://CRAN.Rproject.org/package=readr (2018).

55. Wickham, H., François, R., Henry, L. \& Müller, K. dplyr: A Grammar of Data Manipulation. R package version 0.8.3. https://CRAN.R-project.org/package=dplyr (2018). 
56. Xie, Y. et al. DT: A wrapper of the JavaScript library 'DataTables'. R package version 0.10. https://CRAN.R-project.org/package=DT (2019).

57. Wickham, H. et al. ggplot2: Create elegant data visualisations using the grammar of graphics. $\mathrm{R}$ package version 3.2.1. https://CRAN.R-project.org/package=ggplot2 (2019).

58. Chamberlain, S., Zhu, H., Jahn, N., Boettiger, C. \& Ram, K. rcrossref: Client for various 'CrossRef' 'APIs'. $\mathrm{R}$ package version 0.9.2. https://CRAN.R-project.org/package=rcrossref (2019).

59. Gifi, Albert. Nonlinear Multivariate Analysis. (Wiley, New York, 1991).

60. Mair, P. \& Leeuw, J. D. homals: Gifı methods for optimal scaling. R package version 1.0.9. https://CRAN.R-project.org/package=homals (2021).

61. Dray, S. et al. ade4: Analysis of ecological data: exploratory and euclidean methods in environmental sciences. R package version 1.7.16. https://CRAN.R-project.org/package=ade4 (2020).

62. Husson, F., Josse, J., Le, S. \& Mazet, J. FactoMineR: Multivariate exploratory data analysis and data mining. R package version 2.4. https://CRAN.R-project.org/package=FactoMineR (2020).

63. Kassambara, A. \& Mundt, F. factoextra: Extract and visualize the results of multivariate data analyses. R package version 1.0.7. https://CRAN.R-project.org/package=factoextra (2020).

64. Akaike, H. A new look at the statistical model identification. IEEE Trans. Autom. Control 19, 716-723 (1974).

Figures
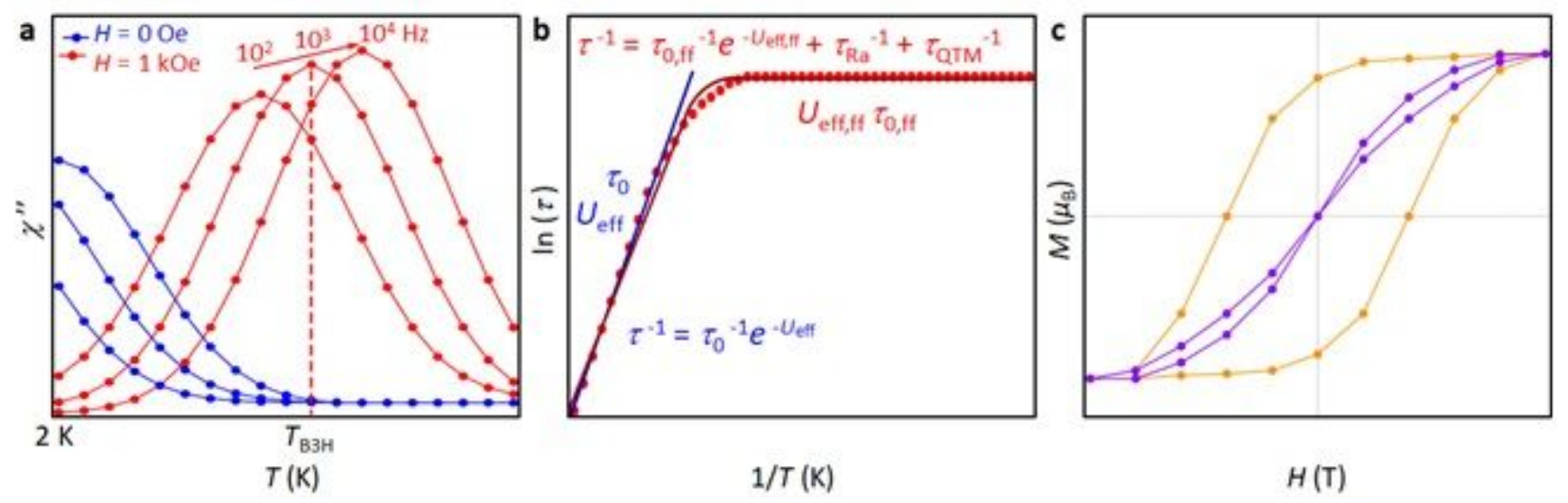

Figure 1

Main magnetic concepts employed in this study. Slow relaxation of the magnetisation in SIMs can manifest in different ways. a, The blocking temperature TB is often characterised by the temperature- or frequency-dependent out-of-phase ac susceptibility $\chi^{\prime \prime}$. At a given frequency, $\chi^{\prime \prime}$ does not necessarily present a maximum; an external magnetic field facilitates this effect by cancelling quantum tunnelling of the magnetisation (QTM). In this study we register the temperature of this maximum as TB3H (TB3), the blocking temperature at $103 \mathrm{~Hz}$ in presence (in absence) of a magnetic field. b. These relaxation dynamics have most often been modelled as an Orbach process, using the Arrhenius equation. The 
effective energy barrier for spin reversal is parameterised as Ueff, and the inverse of the relaxation rate at the high temperature limit is denoted as $\tau 0$. A "full fit" considering other processes in addition to Orbach, such as the Raman process or QTM, in principle results in more accurate values, which are denoted as Ueff,ff, $\mathrm{\tau} 0, \mathrm{ff}$. c, The best metric for slow relaxation is Thyst, the highest temperature at which the system presents magnetic hysteresis (orange). Often, the hysteresis curve is "pinched" (purple), signalling a fast relaxation at zero magnetic field.

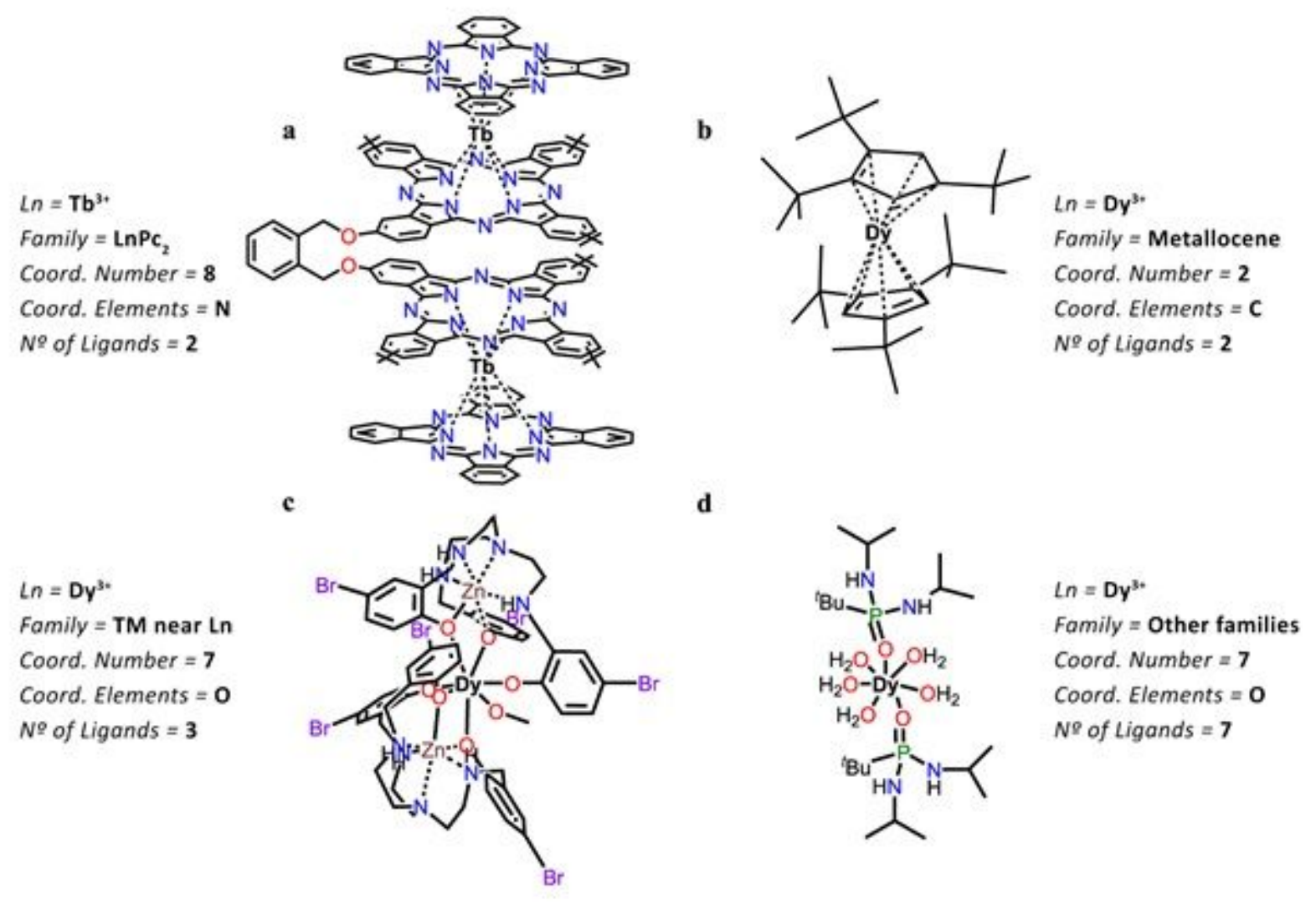

\section{Figure 2}

Molecular structures of some lanthanide-based SIMs from different representative chemical strategies and some of their chemical descriptors. a, Pc "double deckers", abbreviated as LnPc2 (Thyst = $31 \mathrm{~K}$ ). 18 $P c$ complexes, especially the ones where one of the Pc ligands is oxidized, display an $S=1 / 2$ radical and have presented good properties, 19 inspiring many works centered on Pc and/or radical ligands. b, LnCp*2 (Thyst $=60 \mathrm{~K}$ ).20 Metallocene complexes, where the ligands are either cyclopentadienyl or cyclooctatetraene derivatives, have given rise to a series of world records.20-22 c, [Zn2DyL2(MeOH)]- (L is a tripodal ligand, 2,2',2"-(((nitrilotris(ethane-2,1-diyl))tris(azanediyl))tris(methylene))tris-(4bromophenol) $)($ Thyst $=11 \mathrm{~K}) .23$ The introduction of a diamagnetic TM ion near the lanthanide was considered a success23 and has been pursued extensively. d, [L2Dy(H2O)5]3+ ( $L=t B u P O(N H i P r) 2)$, a complex outside the main categories of the present study, which was classified as "other families" (Thyst $=30 \mathrm{~K}) .24$ No single chemical strategy has dominated in terms of reported examples, although many approaches have been paradigmatic (e.g. the use of radicals25,26 and diketonates27). 


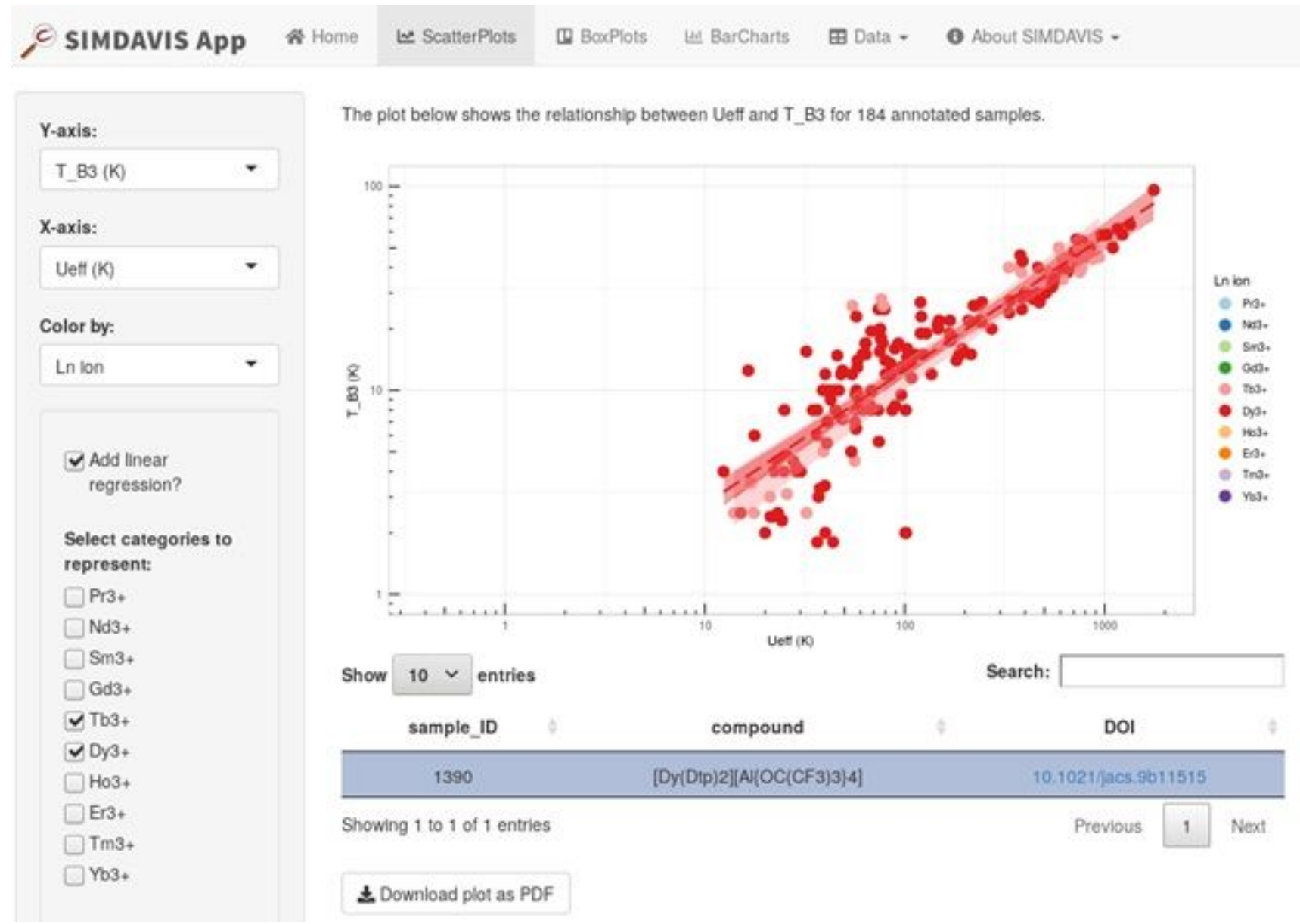

Figure 3

Screenshot of the SIMDAVIS dashboard. It shows an example plot employing the "ScatterPlots" tab, where the users can represent 9 quantitative physical properties versus another in logarithmic scales, as well as a chemical qualitative variable from a dropdown menu, which contains 12 qualitative categorization possibilities; each data point is identified by a color corresponding to its category. This permits the interactive exploration of hundreds of potential magnetostructural correlations between chemical variables, measured experimental values and parameters fitted from physical measurements. In the example, TB3 is represented vs Ueff. Checkboxes were used to add a linear regression for each category and to hide all metal ions except for Tb3+ and Dy3+. This visual estimate on the relation between descriptors of the magnetic behaviour may uncover trends for specific qualitative variables. At any time, the chosen plots can be downloaded as vectorial PDF files. In the example, the data point with the highest TB3 was clicked to display its sample ID, compound name and DOI linking to the article, facilitating further analysis. 

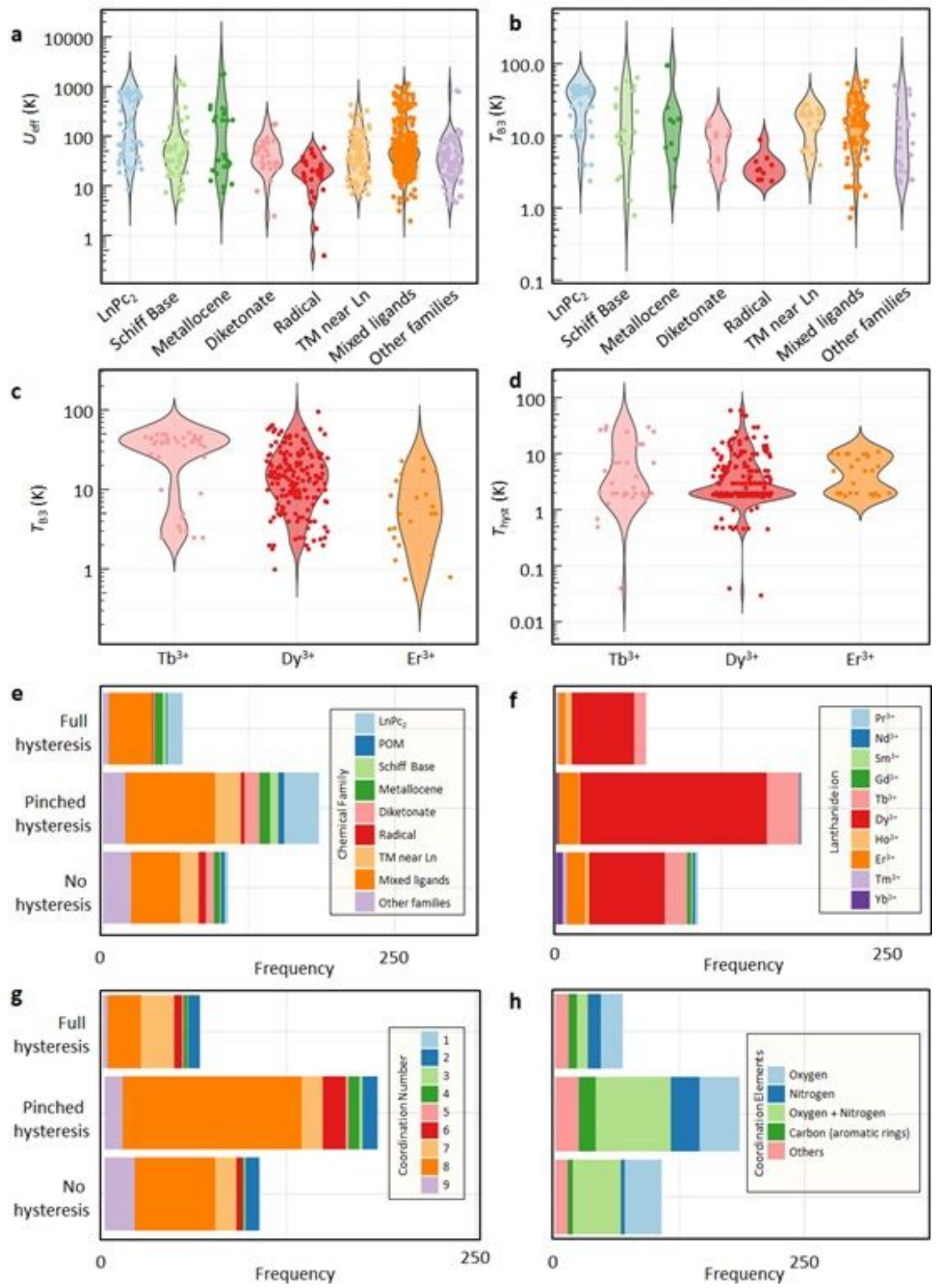

Figure 4

Violin plots and bar charts relating magnetic relaxation behaviour with the main chemical parameters. a, Ueff vs chemical family. b, TB3 vs chemical family. c, TB3 for Tb3+, Dy3+, Er3+. d, Thyst for Tb3+, Dy3+, Er3+. e,f,g,h, Magnetic hysteresis behavior for different chemical families (e), lanthanide ions (f), coordination numbers $(\mathrm{g})$, and elements in the coordination sphere $(\mathrm{h})$. 

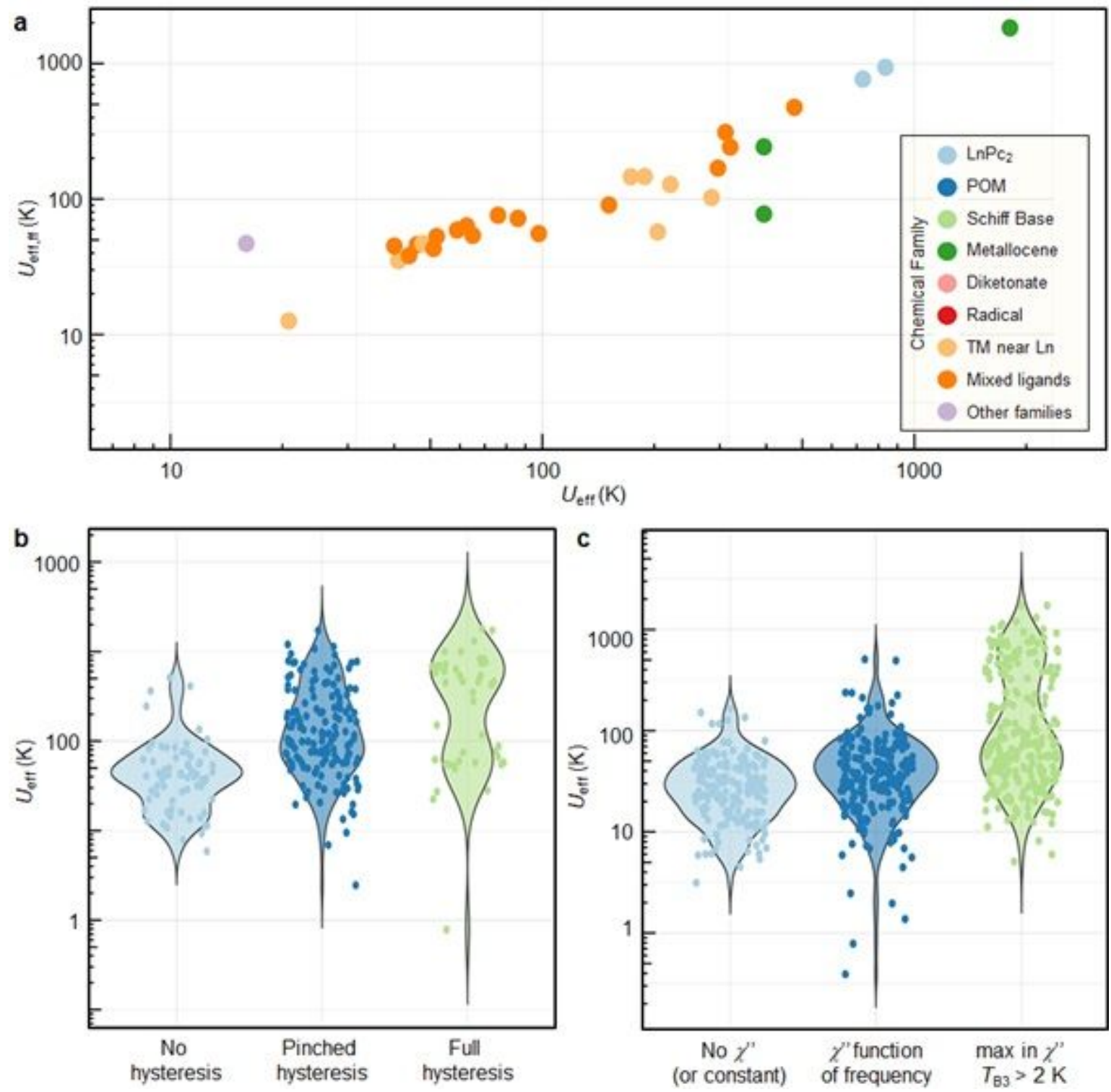

Figure 5

Main dependencies between the physical variables. a, Dependence between Ueff and Ueff,ff. b, Distribution of Ueff for samples depending on their qualitative behaviour in terms of magnetic hysteresis, c, Distribution of Ueff for samples depending on their qualitative behaviour in terms of TB3, or, in its absence, of a frequency-dependent out-of-phase signal “". For a more complete analysis, see Supplementary Sections 5.1 and 6. 
a

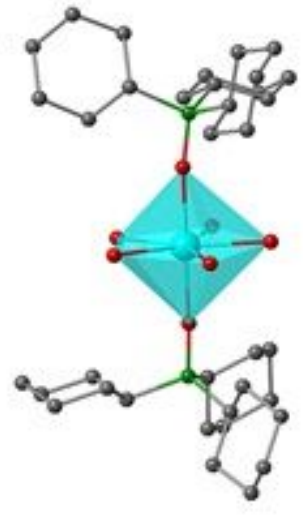

$T_{83}=32 \mathrm{~K}, U_{\text {eff }}=543 \mathrm{~K}, T_{\text {pyst }}=20 \mathrm{~K}$

d

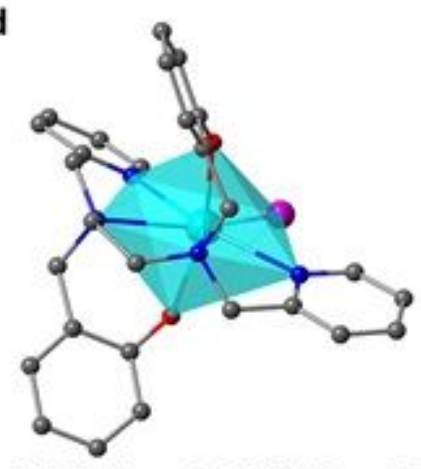

$T_{B 3}=58 \mathrm{~K}, U_{e f f}=1025 \mathrm{~K}, T_{\text {hpse }}=14 \mathrm{~K}$

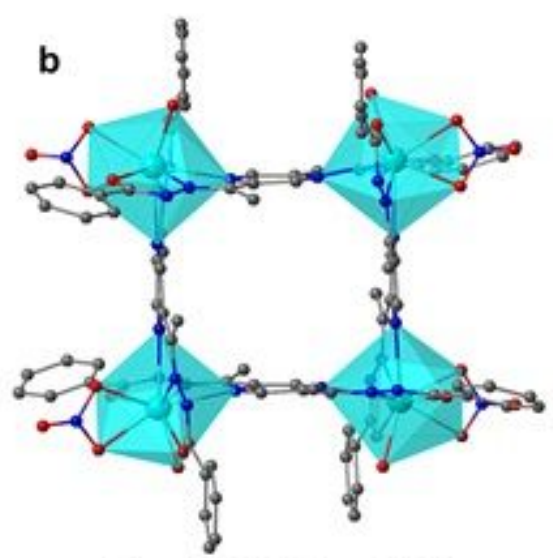

$U_{\text {eff }}=61.3 \mathrm{~K}, T_{\text {hyst }}=16 \mathrm{~K}$

e

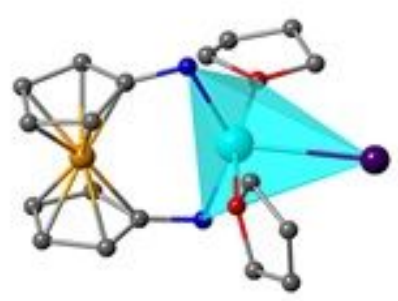

$T_{\text {B3 }}=54 \mathrm{~K}, U_{\text {eff }}=770.8 \mathrm{~K}, T_{\text {hyst }}=14 \mathrm{~K}$ c

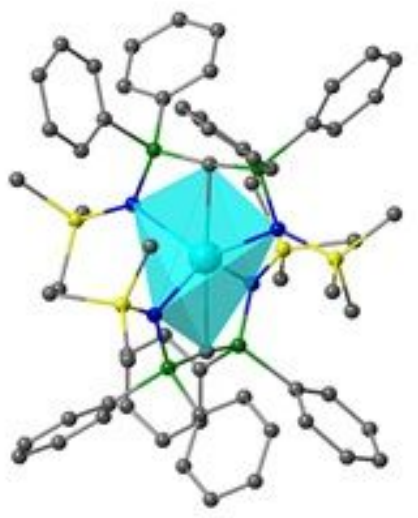

$$
T_{83}=39 \mathrm{~K}, T_{\text {hyst }}=17 \mathrm{~K}
$$

$\mathbf{f}$

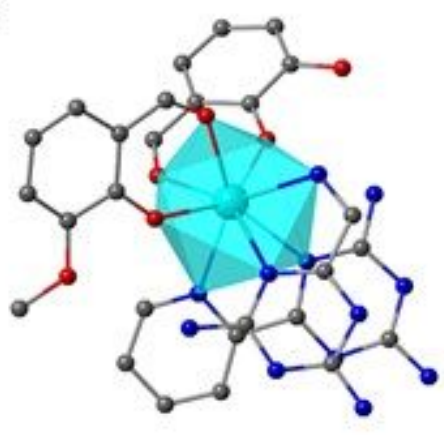

$U_{\text {eff }}=696 \mathrm{~K}, T_{\text {byst }}=12 \mathrm{~K}$

\section{Figure 6}

Promising systems for the development of new high-Thyst SIMs, all chemically distinct from each other and from the TbPc2 and metallocene categories.37,46-50 a, [Dy(Cy3PO)2(H2O) 5$] 3+($ Cy3PO = tricyclohexyl phosphine oxide);37 b, [Dy4(bzhdep-2H)4(H2O)4(NO3)4] (bzhdep = pyrazine-2,5-diylbis(ethan-1-yl-1-ylidene)-di-(benzohydrazide));46 c, [Dy(BIPMTMS)2]- (BIPMTMS = $\{C(P P h 2 N S i M e 3) 2\} 2-) ; 47$ d, [Dy(bbpen)Br] (H2bbpen = N,N'-bis(2-hydroxybenzyl)-N,N'-bis(2methylpyridyl)ethylenediamine);48 e, (NNTBS)Dyl(THF)2 (NNTBS = fc(NHSitBuMe2)2, fc = 1,1'ferrocenediyl);49 f, [DyLz2(o-vanilin)2]+ (Lz = 6-pyridin-2-yl-[1,3,5]triazine-2,4-diamine).50 (Color scheme for atoms: green, $\mathrm{P}$; cyan, Dy; gray, C; blue, N; yellow, Si; orange, Fe; red, O; magenta, Br; purple, I. Hydrogen atoms are not shown for clarity.)

\section{Supplementary Files}

This is a list of supplementary files associated with this preprint. Click to download.

- GraphicalAbstract.png

- Duanetal2021SINatChem.pdf 